\title{
A Supernova at $z=0.458$ and Implications for Measuring the Cosmological Deceleration
}

\author{
S. Perlmutter ${ }^{1,2}$, C. R. Pennypacker ${ }^{1,2}$, G. Goldhaber ${ }^{1,2}$, A. Goobar ${ }^{2,3}$, R. A. Muller ${ }^{2}$, \\ H. J. M. Newberg ${ }^{1,2,4}$, J. Desai², A. G. Kim¹, M. Y. Kim² , I. A. Small ${ }^{1,2}$, B. J. Boyle ${ }^{5}$, \\ C. S. Crawford ${ }^{5}$, R. G. McMahon ${ }^{5}$, P. S. Bunclark ${ }^{6}$, D. Carter $^{6}$, M. J. Irwin ${ }^{6}$, \\ R. J. Terlevich ${ }^{6}$, R. S. Ellis ${ }^{7}$, K. Glazebrook ${ }^{7}$, W. J. Couch ${ }^{8}$, J. R. Mould ${ }^{9}$, T. A. Small ${ }^{9}$, \\ and R. G. Abraham ${ }^{10}$
}

Received

accepted

Astrophysical Journal Letters, 440, L41 (1995).

\footnotetext{
${ }^{1}$ Center for Particle Astrophysics and Space Sciences Laboratory, U.C. Berkeley, California 94720

${ }^{2}$ Lawrence Berkeley Laboratory, Berkeley, California 94720

${ }^{3}$ Swedish Natural Science Research Council (NFR) Fellow

${ }^{4}$ Fermilab, Batavia, Illinois 60510

${ }^{5}$ Institute of Astronomy, Cambridge, United Kingdom

${ }^{6}$ Royal Greenwich Observatory, Cambridge, United Kingdom

${ }^{7}$ University of Durham, Durham, United Kingdom

${ }^{8}$ University of New South Wales, Sydney, Australia

${ }^{9}$ California Institute of Technology, Pasadena, California 91125

${ }^{10}$ Dominion Astrophysical Observatory, Victoria, B.C., Canada
} 


\begin{abstract}
We have begun a program to discover high-redshift supernovae $(z \approx$ 0.25-0.5), and study them with follow-up photometry and spectroscopy. We report here our first discovery, a supernova at $z=0.458$. The photometry for this supernova closely matches the lightcurve calculated for this redshift from the template of well-observed nearby Type Ia supernovae. We discuss the measurement of the deceleration parameter $q_{0}$ using such high-redshift supernovae, and give the best fit value assuming this one supernova is a normal, unextincted Type Ia. We describe the main sources of error in such a measurement of $q_{0}$, and ways to reduce these errors.
\end{abstract}

Subject headings: cosmology: distance scale, dark matter — supernovae: general, SN1992bi 


\section{Introduction}

For over 25 years Type I and Type Ia supernovae (SNe Ia) have been studied as potential "standard candles" for distance measurements (for a review, see Branch \& Tammann 1992). Recently, Branch \& Miller (1993) and Vaughan et al. (1994) emphasized the narrow distribution of absolute magnitudes at maximum light for the subset of SNe Ia that have well-measured lightcurves, and are not unusually red or spectroscopically peculiar. This subset of 27 "normal" SN Ia has a dispersion, $\sigma_{M_{B}}=0.3$, that is completely accounted for by measurement errors, so the intrinsic dispersion should be smaller still. Sandage \& Tammann (1993) estimated $\sigma_{M_{B}}^{\text {intrinsic }}<0.2$ by simulating the effects of intrinsic dispersion on Malmquist bias (using a slightly different set of SNe).

Phillips (1993) noted a correlation between absolute magnitude and lightcurve decay time in 9 well-studied SNe Ia (including a few "peculiars"). Magnitude corrections based on such a correlations can "sharpen up" the standard candle by calibrating it, thus improving on the current relatively narrow dispersion and also making it possible to include some peculiar SNe. For this paper, however, we will take the intrinsic dispersion without this

calibration to be $\sigma_{M_{B}}^{\text {intrinsic }} \approx 0.25$, and we will take the error in the mean absolute magnitude for the $27 \operatorname{good}$ SNe Ia to be $\sigma_{M_{B}}^{\text {mean }}=\sigma_{M_{B}} / \sqrt{27} \approx 0.06$ (note that these are somewhat more conservative estimates that those of Sandage \& Tammann (1993)).

Type Ia's are on average the brightest SNe and therefore could be used to measure large cosmological distances and, in particular, the deceleration parameter $q_{0}$ of the expanding universe (Tammann 1979; Colgate 1979). With many observable features in their spectra and their lightcurves, SNe Ia have an advantage as standard or calibrated candles because they can be checked one-by-one for evolutionary differences at high redshift. For example, the lightcurve decay time or the blueshift of spectral features may indicate explosion strength, and can be checked for systematic differences from nearby supernovae. 
To use SNe as tools for cosmology, we have developed a strategy to find and study high-redshift SNe systematically, using new wide-field, high-resolution CCD cameras and image analysis techniques that provide the rapid response time necessary to follow these short-lived events (Perlmutter et al. 1993). In 1992 March and April, we demonstrated this strategy using the Isaac Newton 2.5 meter Telescope (INT) on La Palma, and found a SN at $z=0.458$. The farthest previously detected SN was found at $z=0.31$ in the SN search of Norgaard-Nielsen et al. (1989).

\section{Observations and Data Reduction}

During 1992 March 24 - 28, we observed 54 high-galactic latitude fields, 43 of them centered on high-redshift clusters, using 10-minute $R$ band exposures in seeing better than 2 arcsec. Approximately 200 galaxies were visible in the redshift range $z=0.25-0.5$ in each

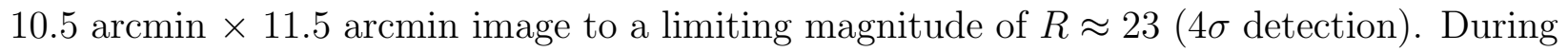
1992 April 21 - May 2 the same fields were re-observed, in pairs of 7-minute exposures offset by $\sim 6$ arcsec to facilitate rejection of cosmic rays and CCD defects. Following each night's observations we used semi-automated image analysis software to search for new point-sources that had appeared since the reference images were taken.

With good seeing and 0.57 arcsec pixels, we were able to distinguish candidate SNe from other variable sources (e.g. QSO's) by looking for a resolved host galaxy. The time between observations at the INT and the completion of analysis in Berkeley was typically less than 2 days, so interesting candidates could trigger follow-up observations. The best of these SN candidates (SN 1992bi) was found at R.A. $=16^{h} 8^{m} 28^{\mathrm{s}} .4$, Dec. $=+39^{\circ} 54^{\prime} 58^{\prime \prime}$ (equinox 1950.0), 1".5 east and 0.5 north from the core of an $R \approx 21.3$ magnitude galaxy (Pennypacker, et al. 1992). We observed the candidate on 5 more nights during the 2.5 weeks after discovery and on 4 nights during the 9 months after it had faded below detectability. Figure 1 (Plate L\#) shows the images of the host galaxy before, during, 
and after the event. To the right of each image is the same image after subtracting off a "reference" image from Day 103 of the host galaxy alone.

The subtraction images of Figure 1 were used to measure the photon flux $f_{R}^{92 b i}$ of the SN candidate on each observation. The Day 396 flux is taken as the best estimate of the host galaxy light and is subtracted out of each $f_{R}^{92 b i}$ measurement. The night-to-night "relative" measurement error is approximately $9 \%$ at maximum light, and is primarily due to photon noise of the background sky. A smaller error contribution, 5\% at maximum light, is due to slight mismatches of the two images before subtraction of the host galaxy reference image from the measured image of the galaxy plus SN. These errors were determined by extensive testing of the plausible range of matching parameters (position offset, seeing, and transmission ratio) for their effect on the resulting magnitude measurements. To obtain the absolute measurement error we must add (in quadrature with these relative errors) an overall error due to the sky noise of the Day 396 reference image, amounting to 13\% near peak. Table 1 lists for each observation the flux $f_{R}^{92 b i}$ and relative error of the SN candidate measured from the subtraction images (note that $f_{R}^{92 \mathrm{bi}}$ can be negative due to photon noise of the subtracted host galaxy and subtracted sky).

The calibration to $R$ magnitude was made on day 149 using the INT/EEV5 camera to image M92 and the SN field consecutively (repeated 3 times to check photometric stability) with the same airmass within 0.02 . These observations were repeated in the $V$ and $I$ bands. Our relative photometry agreed with the calibration of 23 stars in M92 (Christian et al. 1985, revised L. Davis, private communication) with a root-mean-square error of 0.005 mag, and yielded the following calibration: $m_{R}^{92 \mathrm{bi}}=-2.5 \log f_{R}^{92 \mathrm{bi}}+28.20$, where $f_{R}^{92 \mathrm{bi}}$ is measured in units normalized to the photoelectrons/minute observed at the INT 2.5-meter on Day 103. The uncertainty in transferring this standard to each individual night's observation is $\sigma_{c a l}=0.02$ mag, primarily due to the photon noise on $\sim 20$ bright stars in the SN field found 
in both the standard image and each night's images. (Note that the first order extinction is calibrated out in this procedure, and that the extinction color dependence is negligible, because the calibration observations were in the $R$ band and at an airmass within 0.2 of each of the SN observations. The color term is $<0.01$ implying that our instrumental $r$ band is quite close to the calibration's Cousins $R$.)

Twelve attempts to obtain the SN spectrum at four observatories around the world were all unsuccessful due to weather (and one instrument failure). On 1992 August 29, we obtained two 1800-second spectra of the host galaxy using the Low Dispersion Survey Spectrograph 2 on the William Herschel 4.2-meter telescope, and determined the redshift $z=0.458 \pm 0.001$. Both spectra show a strong emission line at 5437 Angstroms. Identifying this line as $[\mathrm{OII}] \lambda 3727$ at $z=0.458$ allows the weak absorption features longwards of it to be identified with $\mathrm{Ca} \mathrm{H}, \mathrm{Ca} \mathrm{K}$, and $\mathrm{H} \delta$ (see Figure 2).

\section{Analysis}

Traditionally one compares photometry of high-redshift objects to photometry of nearby objects by calculating a $K$-correction to account for the different parts of the spectrum that fall in one given filter band for objects at different redshifts. A more robust procedure in this case is to compare the photometry of Table 1 to the apparent $R$-band lightcurve, $m_{R}(t)$, calculated for $z=0.458$ from the standard lightcurve of well-observed nearby SN Ia in the $B$-band. This standard lightcurve, $M_{B}(t)$, is an apparent magnitude versus redshift measurement and thus implicitly depends on the Hubble constant chosen. However, the expression $\left[M_{B}(t)-5 \log H_{0}\right]$ that will appear bracketed together throughout this Letter is independent of the choice of Hubble constant, as is the resulting measurement of $q_{0}$. Since events seen at $z=0.458$ last 1.458 times as long as they do locally, this lightcurve term must be time dilated to $\left[M_{B}(t /(1+z))-5 \log H_{0}\right]$. The calculated distant $R$-band lightcurve (for $\Lambda=0$ ) is thus: 


$$
\begin{aligned}
m_{R}(t)=[ & \left.M_{B}(t /(1+z))-5 \log H_{0}\right]+\Delta m_{R B}+A_{R}+25 \\
& +5 \log \left(c q_{0}^{-2}\left[1-q_{0}+q_{0} z+\left(q_{0}-1\right)\left(2 q_{0} z+1\right)^{\frac{1}{2}}\right]\right),
\end{aligned}
$$

where $A_{R} \simeq 0.006$ is the $R$ extinction in our Galaxy at $(l, b)=(63.27,47.24)$ (Burstein \& Heiles 1982; Burstein, private communication). The $\Delta m_{R B}$ term is the analog of the traditional $K$-correction:

$$
\begin{aligned}
\Delta m_{R B} & =-2.5 \log \left(\frac{\int F_{\lambda}^{A 0 V}(\lambda) S_{B}(\lambda) d \lambda}{\int F_{\lambda}^{A 0 V}(\lambda) S_{R}(\lambda) d \lambda} \frac{\int F_{\lambda}^{S N}(\lambda /(1+z)) S_{R}(\lambda) d \lambda}{\int F_{\lambda}^{S N}(\lambda) S_{B}(\lambda) d \lambda}\right)+2.5 \log (1+z) \\
& =-2.5 \log \left(\frac{\int F_{\lambda}^{A 0 V}(\lambda) S_{B}(\lambda) d \lambda}{\int F_{\lambda}^{A 0 V}(\lambda) S_{R}(\lambda) d \lambda} \frac{\int F_{\lambda}^{S N}\left(\lambda^{\prime}\right) S_{R}\left(\lambda^{\prime}(1+z)\right) d \lambda^{\prime}}{\int F_{\lambda}^{S N}(\lambda) S_{B}(\lambda) d \lambda}\right)
\end{aligned}
$$

where $S_{B}(\lambda)$ and $S_{R}(\lambda)$ are the response functions in the $B$ and $R$ bands, and $F_{\lambda}^{S N}(\lambda)$ and $F_{\lambda}^{A 0 V}(\lambda)$ are the flux per unit of wavelength of the supernova and the standard AOV star SED for which $B=R=0$ in the Landolt system.

The correction $\Delta m_{R B}$ accounts for the different zero points of the $R$ and $B$ magnitude systems, and for the the redshifting of the SN spectrum. We calculate $\Delta m_{R B}=-0.7 \pm 0.05$ using numerical integration with spectra of 3 SNe Ia with overlapping epochs from 15 days before to 15 days past maximum (in the SN rest frame). The error represents the variation for a given $\mathrm{SN}$ over time; the $\mathrm{SN}$-to-SN variation at maximum is $0.02 \mathrm{mag}$. The relatively small variation of $\Delta m_{R B}$ over time is due to the approximate match at $z=0.458$ between the observer's $R$ response function and the SN rest-frame's $B$ response function, i.e. $S_{R}(\lambda(1+z)) \approx S_{B}(\lambda)$. At $z=0.458, \Delta m_{R B}$ is therefore less sensitive to the precise knowledge of the changing SN spectrum, an advantage over the usual $K$-correction. (The calculation of $\Delta m_{R B}$ was checked by reproducing the $K$-corrections of Hamuy et al. (1993) to within $0.001 \mathrm{mag}$ for the $3 \mathrm{SNe}$.)

Taking $\left[M_{B}\left(t_{\max }\right)-5 \log \left(H_{0} / 75\right)\right]=-18.86 \pm 0.06$, where the error is the error in the mean of the distribution as discussed above (Branch and Miller 1993, Vaughan et al. 1994), and the template standard $B$ lightcurve, $M_{B}(t)-M_{B}\left(t_{\max }\right)$, of Leibundgut (1991 
and private communication), we calculate the lightcurves shown in Figure 3 for two values of the deceleration parameter, 0 and 0.5 . At $z=0.458$ a standard candle is 0.25 mag fainter (for $\Lambda=0$ ) in an empty universe, $q_{0}=0$, than in a critically closed universe, $q_{0}=0.5$.

Figure 3 also shows the measured photometry data from Table 1 . When we perform a $\chi^{2}$ fit of the the data points of Table 1 to the calculated lightcurve, $f_{R}(t)$, varying the values of $q_{0}$ and the time offset, $\Delta t$, we find the best fit at $q_{0}=0.07$. If there is significant host galaxy extinction then $q_{0}$ would be larger. The individual error bars shown in Figure 3 are the relative errors used in the fit, while the errors from the reference image photometry and calibration contribute to the overall error bar $\sigma_{c}$ (discussed below). Note that the preand post-SN measurements constrain the time-offset fit.

Although the photometry data shown in Figure 3 are clearly consistent with the lightcurve of an SN Ia at $z=0.458$, several alternative identifications were considered. It is very unlikely (Prob $<1 \%$ ) that this event is a projected foreground variable star in our Galaxy or a projected background quasar or AGN. The lack of repeated flare events makes these identifications still more unlikely. The data are also consistent with an unusually bright SN IIL. Assuming the currently favored cosmologies with $q_{0}<2$ (or, equivalently, $H_{0}>40 \mathrm{~km} \mathrm{sec}^{-1} \mathrm{Mpc}^{-1}$ and $\tau_{\text {universe }}>12$ Gyr; see Carroll, Press, \& Turner 1992), we can estimate the relative probability of a SN IIL identification as follows: First, we note that the event's absolute magnitude could not be more than 0.7 magnitudes fainter than the SN Ia value $M_{B}\left(t_{\max }\right)$ used above, because $q_{0}$ would then have to be $>2$ to fit our photometry data. We then compare, in nearby spiral galaxies, the number of SNe Ia and the number of SNe IIL brighter than this minimum: $M_{\min } \equiv M_{B}\left(t_{\max }\right)+0.7+0.3$ (the additional 0.3 magnitude allows for measurement error). The result is $\sim 10 \mathrm{SNe}$ Ia for every SN IIL (see Miller \& Branch 1990), and we use this ratio as the relative probability of these identifications. This is, of course, a rough approximation that assumes that the 
SN detection efficiency falls off towards fainter absolute magnitudes at least as fast at $z=0.458$ as in nearby searches. Similarly, we estimate $<1 / 30$ for the relative probability that this event is a peculiar SN Ia or one of the SNe Ia showing clear evidence of extinction, since over 30 times as many normal, unextinguished SNe Ia brighter than $M_{\min }$ are found in nearby galaxies (Branch \& Miller 1993). The identification of this event as a normal SN Ia at $z=0.458$ is therefore made with $\sim 90 \%$ confidence level (for any $q_{0}<2$ ) for the purposes of this paper. The confidence level would be greater (and the assumption of $q_{0}<2$ unnecessary) if spectra or color photometry were available.

The uncertainty in this measurement of $q_{0}$ has two major contributory sources. The first source is the uncertainty, $\sigma_{R} \approx 0.14$, in the apparent $R$ magnitude at maximum light of the $z=0.458 \mathrm{SN}$. This includes the photon noise and image-matching uncertainty in the images observed near maximum light, $\sigma_{\text {peak }} \approx 0.06$, and the photon noise and calibration uncertainty in the Day 396 reference image used to subtract off the host galaxy light, $\sigma_{r e f} \approx 0.13$. (A better reference image will reduce this error source significantly.)

The second and much larger contributory source of measurement error enters in when this distant photometry is compared with the nearby apparent magnitude-Hubble velocity relation, i.e. the nearby measurement of $\left[M_{B}-5 \log H_{0}\right]$. The uncertainty in the mean of this quantity may be as small as $\sigma_{M_{B}}^{\text {mean }} \approx 0.06$, but since we do not know where in the $M_{B}$ distribution our particular SN at $z=0.458$ lies, we must add in quadrature an error contribution for the intrinsic dispersion $\sigma_{M_{B}}^{\text {intrinsic }} \approx 0.25$. This is currently the dominant source of error. Since it is plausible that this is an overestimate of the intrinsic dispersion, as discussed above, we will separate out this error in the subsequent analysis, and define a $\sigma_{o}$ to include only the other sources of error. Finally, we also include in quadrature an error of $\sigma_{R B} \approx 0.05$ for the uncertainty in $\Delta m_{R B}$, so $\sigma_{o}^{2}=\sigma_{R}^{2}+\sigma_{M_{B}}^{\text {mean2 }}+\sigma_{R B}^{2}$.

The total error in the distant SN apparent magnitude and in the comparison with the 
nearby apparent magnitude-velocity relation is $\sigma_{m a g}= \pm \sigma_{o} \pm \sigma_{M_{B}}^{\text {intrinsic }}= \pm 0.16 \pm 0.25$. (Figure 3's $\sigma_{c}$ is the equivalent total error in flux.) This propagates through to an uncertainty on the $q_{0}$ measurement of $\sigma_{q_{0}} \approx \sigma_{m a g} / z$, yielding a measurement of $q_{0}=0.1 \pm 0.3 \pm 0.55$ (for $\Lambda=0$ ). This measurement is stricter in its lower limit than its upper limit, since host galaxy extinction would increase $q_{0}$. We emphasize again the assumption of a normal SN Ia.

\section{Discussion}

We wish to stress two main results:

(1) From a single SN at $z=0.458$ known to be a "normal" type Ia, we could in principle measure $q_{0}$ with an uncertainty comparable to the previous measurements in the literature that required larger numbers of objects (galaxies or clusters) and needed significant corrections for evolutionary effects (for reviews, see Rowan-Robinson 1985 and Sandage 1988). Clearly the measurement of $q_{0}$ cannot rest on a single distant SN Ia, and in this particular case we do not have the color photometry or the spectra that would enable us to screen for host galaxy extinction and peculiar SNe. We are continuing to search for

distant SNe Ia, and we are scaling up the detector size and sensitivity so that we can find many high-redshift SNe Ia, and follow their lightcurves, colors and spectra over maximum light. This will make it possible to compare the luminosity function distribution of distant and nearby SNe, and to identify and reject extinguished SNe.

(2) The measurement errors that contribute to the uncertainty in $q_{0}$ can all be reduced. The dominant error is due to both our uncertainty in the true width of the SN Ia absolute magnitude distribution and our uncertainty in where a given distant SN Ia falls in this distribution. As a few more SN Ia past Coma are discovered and studied, we may find that the distribution width is smaller than can be seen in the nearby SNe where peculiar velocities can mask the Hubble expansion. Hamuy et al. (1994) are working on such a search, and the Berkeley Automated Supernova Search (Muller et al., 1992), now being 
moved to a good astronomical site, may also find and study these SNe.

Alternatively, if the dispersion is in fact $\sigma_{M_{B}}^{\text {intrinsic }}=0.25$ then discovering $N$ more 
distant SNe Ia will make it possible to statistically reduce this error by $\sim \sqrt{N}$. The main source of concern here is that the distant $M_{B}$ distribution should be the same as the nearby distribution for the comparison to be valid; it will be important to ensure that the distant SN detection limit be significantly fainter than the peak of the $M_{B}$ distribution at the redshifts searched to avoid Malmquist-bias distortion of the shape of the distribution, particularly if there are rare superluminous Type Ia's (see the discussion of the possibly superluminous SN 1991T in Ford et al. 1993).

The uncertainty in the $K$-correction (in this case, $\Delta m_{R B}$ ) is the only remaining source of error that cannot be improved simply by using longer exposure times, larger telescopes, or darker sites. It therefore may be useful to measure the lightcurves for future nearby SNe Ia using filter sets designed to give "blue-shifted $R$ " bands for a sampling of values of $z$. Together with SNe Ia spectra to interpolate between these $z$ values, these "blue-shifted $R$ " lightcurves should allow accurate determinations of the $K$-corrections.

It will be necessary to check the effects of metallicity and other evolutionary changes on the SN Ia absolute magnitude to validate measurements of $q_{0}$ derived from SNe Ia. Deep spectra of distant SNe will be necessary to look for any evolutionary changes with respect to spectra of nearby SNe. Metallicity effects can be studied by comparing nearby SNe Ia found in different metallicity environments. So far, however, no indications of magnitude evolution have been found observationally, and the standard model of SNe Ia gives theoretical reasons to believe that SN Ia magnitudes should not evolve. Thus, the prospects for obtaining a precise value for $q_{0}$ from SNe Ia appear good.

[Note added in press: This project has by now found 6 additional SNe around $z \sim 0.4$ (e.g., Perlmutter et al 1994). With these it should be possible to use lightcurve decay-time calibration or lightcurve shape calibration, recently shown to yield $\sigma_{M_{B}}^{\text {intrinsic }}$ as small as 0.1 (Hamuy et al. 1994) to 0.2 (Riess et al. 1994).] 
M. Bessell, B. Leibundgut, B. Schmidt, M. Hamuy, and D. Branch kindly supplied unpublished data, spectra, programs, and some results for the calculation of $\Delta m_{R B}$. We thank D.Burstein, K.Chambers, R.Clegg, M.De Robertis, S.Kulkarni, P.Meikle, F.Sanchez, and P.Vilchez for observational efforts. This work has support from the U.S. Dept. of Energy (DE-AC03-76SF000098) and National Science Foundation (ADT-88909616). 


\begin{tabular}{crrr}
\hline $\begin{array}{c}\text { Day } \\
\text { JD-2448707 }\end{array}$ & Telescope & $\begin{array}{r}\text { Exposure } \\
\text { ( Camera }\end{array}$ & $\begin{array}{c}f_{R}^{92 \mathrm{bi}} \\
\left(\text { relative flux) }{ }^{a}\right.\end{array}$ \\
\hline \hline 0 & INT/EEV5 & 600 & $-18 \pm 42$ \\
2 & INT/EEV5 & 600 & $25 \pm 25$ \\
30 & INT/EEV5 & $2 \times 420$ & $260 \pm 21$ \\
34 & INT/EEV5 & $2 \times 420$ & $251 \pm 22$ \\
38 & INT/EEV5 & $2 \times 480$ & $241 \pm 29$ \\
43 & INT/EEV8 & $2 \times 300$ & $213 \pm 23$ \\
45 & INT/EEV8 & 300 & $169 \pm 41$ \\
47 & INT/EEV8 & 300 & $215 \pm 34$ \\
77 & Palomar/CosMIC & 300 & $28 \pm 44$ \\
103 & INT/EEV5 & $5 \times \sim 170$ & $20 \pm 19$ \\
148 & INT/EEV5 & $6 \times 300$ & $12 \pm 22$ \\
330 & INT/Ford & $6 \times 320$ & $-34 \pm 22$ \\
396 & INT/EEV5 & $7 \times 400$ & $\ldots$ \\
\hline
\end{tabular}

Table 1: Observation log and supernova flux

${ }^{a}$ The host galaxy flux from the image of Day 396 has been subtracted off. Relative flux units are normalized to the photoelectrons/minute observed at the INT 2.5-meter on Day 103. The calibration is: $m_{R}^{92 \mathrm{bi}}=-2.5 \log f_{R}^{92 \mathrm{bi}}+(28.20 \pm 0.02)$. The listed relative error includes sky photon noise and image matching error for each individual image. An overall error of 30 units should be added in quadrature to account for the sky noise of the reference image. 


\section{REFERENCES}

Branch, D. \& Miller, D. L. 1993, ApJ , 405, L5

Branch, D. \& Tammann, G. A. 1992, ARA\&A , 30, 359

Burstein, D. \& Heiles, C. 1982, AJ , 87, 1165

Christian, C. A. , Adams, M., Barnes, J. V., Butcher, H. , Hayes, D. S., Mould, J. R.,\& Siegel, M. 1985, PASP , 97, 363

Colgate, S. A. 1979, ApJ , 232, 404

Ford, C. H., Herbst, W., Richmond, M. W., Baker, M. L., Filippenko, A. V., Treffers, R. R., Paik, Y., \& Benson, P. J. 1993, AJ , 106, 1101

Hamuy, M., Phillips, M. M., Wells, L. A., \& Maza, J. 1993, PASP , 105, 787

Hamuy, M., Phillips, M. M., Maza, J., Suntzeff, N.B., Schommer, R., \& Aviles, R. 1994, AJ, in press

Leibundgut, B., Tammann, G., Cadonau, R., \& Cerrito, D. 1991, A\&AS , 89, 537

Miller, D. L. \& Branch, D. 1990, AJ , 100, 530

Muller, R. A., Newberg, H. J. M., Pennypacker, C. R., Perlmutter, S., Sasseen, T. P., \& Smith, C. K. 1992, ApJ , 384, L9

Norgaard-Nielson, H. U., Hansen, L., Jorgensen, H. E., Salamanca, A. A., Ellis, R. S., \& Couch, W. J. 1989, Nature , 339, 523

Pennypacker et al., C. 1992, IAU Circular , 5652 
Perlmutter, S., Pennypacker, C., Goldhaber, G., Muller, R., \& Newberg, H. 1993, Lawrence Berkeley Laboratory Reports, LBL-34128

Perlmutter et al., S. 1994, IAU Circular, 5956

Phillips, M. M. 1993, ApJ , 413, L105

Riess, A. G., Press, W. H., \& Kirshner, R. P. 1994 ApJ, submitted

Rowan-Robinson, M. 1985, The Cosmological Distance Ladder, W. H. Freeman and Company, New York

Sandage, A. 1988, ARA\&A, 26, 561

Sandage, A. \& Tammann, G. A. 1993, ApJ , 415, 1

Tammann, G. A. 1979, in F. D. Machetto, F. Pacini, \& M. Tarenghi (eds.), Astronomical Uses of the Space Telescope, p. 329, ESO, Geneva

Vaughan, T., Branch, D., Miller, D., \& Perlmutter, S. 1994, ApJ , in press

This manuscript was prepared with the AAS LATEX macros v3.0. 


\section{FIGURE CAPTIONS}

Figure 1 (Plate L\#): The left panel of each pair shows the image of the host galaxy with or without the supernova. The time, $t$, is JD - 2448707. The superposed contours start at 22 photoelectrons/minute/pixel, with each additional contour representing 11 photoelectrons/minute/pixel. Note that these images are normalized to the same sky transmission but not matched in seeing. The right panel of each pair shows the same images after subtracting off the host galaxy image of day 103. (Days 0, 330, and 396 are not shown.)

Figure 2: Spectrum of the host galaxy, observed on 1992 August 29 (Day 156) on the William Herschel 4-meter telescope.

Figure 3: Solid curve shows the calculated lightcurve, $f_{R}(t)$, for the best fit $q_{0}=0.1$, based on the template $B$ lightcurve for nearby supernovae. The dotted curves are $f_{R}(t)$ for $q_{0}=0.5$ (upper curve) and for $q_{0}=0$ (lower curve). The photometry points are $f_{R}^{92 \mathrm{bi}}$ from Table 1 (days 330 and 396 not shown). The inner error bar, $\sigma_{c}$, shows the combined uncertainty at maximum light in $f_{R}(t)$ and in the reference image's photometry and

magnitude calibration; the outer error bar includes the intrinsic dispersion, $\sigma_{M_{B}}^{\text {intrinsic }}$. The inset scale shows $R$ magnitudes. For the best fit $q_{0}$, the peak magnitude is $m_{R}=22.2$ on Day 34 .

Fig. 1.- 
Fig. 2.-

Fig. 3.- 

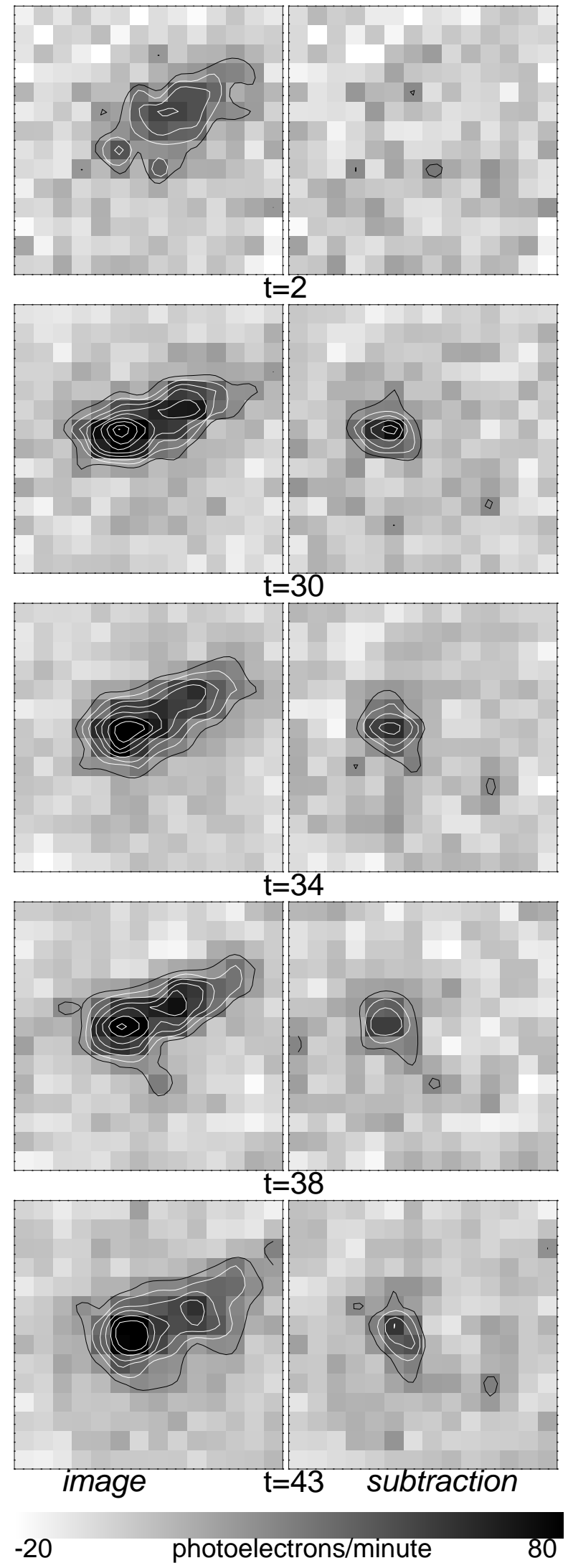
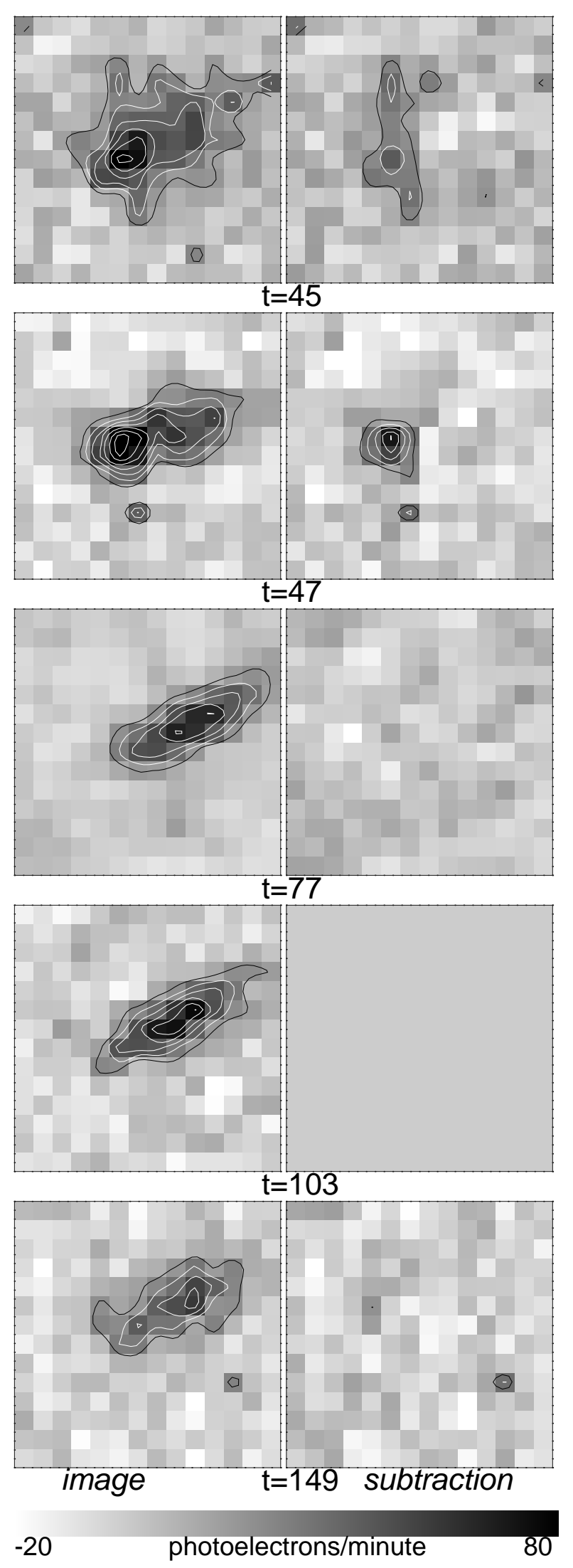

Fig. 1.- 


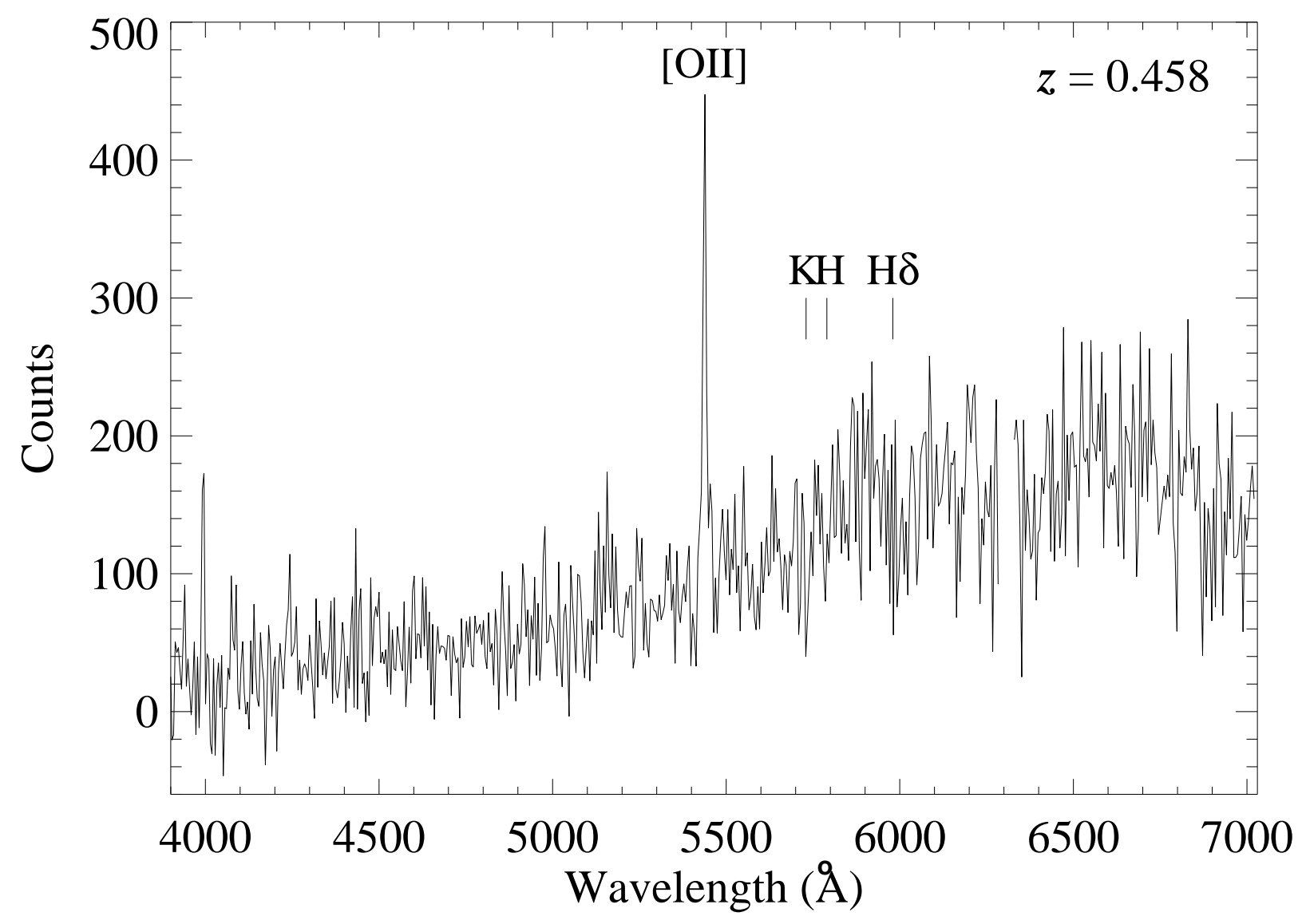

Fig. 2.- 


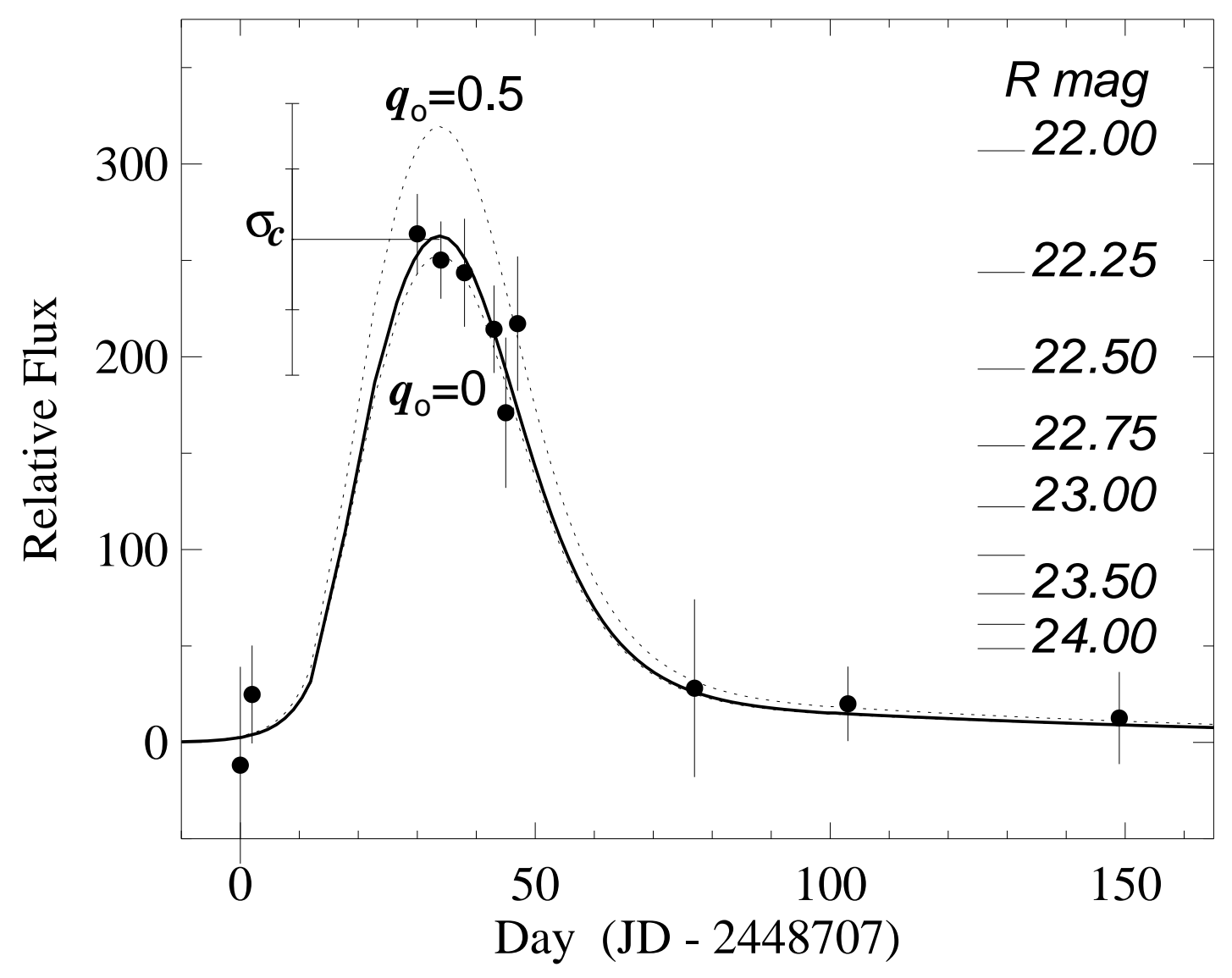

Fig. 3.- 